\title{
Sterbehelfer - eine neue Rolle für Europas Ärzteschaft?
}

Georg Bosshard

Interessenverbindungen des Autors: keine

\section{* Hier wie im folgenden sind jeweils beide Geschlechter angesprochen.}

Korrespondenz:

PD Dr. med. Georg Bosshard

Privatdozent für Klinische Ethik

Institut für Rechtsmedizin

der Universität Zürich

Winterthurerstrasse 190 / Bau 52

CH-8057 Zürich

Tel. 0446355627

bosh@irm.unizh.ch

\section{Einleitung und Terminologie}

Die vorliegende Arbeit analysiert die Rechtslage und insbesondere die ärztlich-standesethischen Richtlinien zur Frage der Rolle des Arztes* bei der Suizidbeihilfe und der aktiven Sterbehilfe auf Verlangen in Westeuropa, zeigt aktuelle Entwicklungen auf und diskutiert deren Bedeutung für die Ärzteschaft.

Im Gegensatz zur Schweiz wird in manchen anderen europäischen Ländern oftmals kein wesentlicher ethischer Unterschied zwischen der Suizidbeihilfe (bei der der Patient sich eine ärztlich verschriebene tödliche Substanz selber verabreichen muss - assistierte Selbsttötung) und der aktiven Sterbehilfe auf Verlangen gesehen (bei der die Verabreichung durch den Arzt erfolgt - Tötung auf Verlangen). Im deutschsprachigen Raum besteht die zunehmende Tendenz, das Wort «Sterbehilfe» in eingeengter Bedeutung primär als Überbegriff für die beiden genannten Praktiken zu verwenden, und genau so ist dieser Begriff auch in der vorliegenden Arbeit zu verstehen. Die sogenannten «passiven» (Behandlungsabbruch und -verzicht) sowie «indirekten» Formen der Sterbehilfe (Opiate und Sedative am Lebensende mit möglicher Lebensverkürzung als Nebenwirkung) sind also nicht das Thema dieses Artikels.

In den letzten zwanzig Jahren hat in fast allen westlichen Staaten eine intensive Diskussion zur Frage der Liberalisierung von Sterbehilfe eingesetzt. Gleichzeitig liess sich in fast allen westeuropäischen Staaten eine zunehmende Akzeptanz von Sterbehilfe in der Allgemeinbevölkerung feststellen. In verschiedenen Ländern kam es in der Folge zu ernsthaften Versuchen, das Strafrecht in diesem Bereich zu öffnen. Bisher war diesen Versuchen allerdings nur in den Niederlanden und Belgien Erfolg beschieden. Daneben hat sich bekanntlich auch in der Schweiz in den letzten zwei Jahrzehnten eine offene Praxis der Suizidbeihilfe herausgebildet, die auf der vorbestehenden offenen Rechtslage in diesem Bereich basiert.

Traditionellerweise hat sich die Ärzteschaft immer von Suizidbeihilfe und aktiver Sterbehilfe distanziert. Seit der geschilderten politischen Debatte wird es aber zunehmend schwierig, diese Distanz einfach durch Hinweis auf die

\section{Aide au décès: un nouveau rôle pour les médecins européens?}

L'approbation envers l'assistance médicale au suicide et l'aide active au décès, pratiquées sur demande, a augmenté dans la plupart des pays européens au cours de ces 20 dernières années. La présente étude analyse la situation juridique et en particulier les directives médicales et déontologiques concernant le rôle du médecin en cas d'aide au décès dans six pays européens.

Contrairement à l'Allemagne, à la Norvège et partiellement à la Grande-Bretagne, les médecins de Suisse et de Belgique ont renoncé à leur refus initial de l'assistance au suicide. Les directives médicales et déontologiques de ces deux pays considèrent aujourd'hui que la réglementation de l'aide au décès est une question d'ordre essentiellement politique et sociétal. Cette position «neutre» se différencie à son tour de la position officielle de la société hollandaise de médecine qui, depuis les années 70 , joue un rôle-clé dans le développement de I'aide active au décès sur demande qui est devenue une forme de «décision médicale en fin de vie».

Une société qui souhaite autoriser par principe I'aide au décès devrait réfléchir avec soin pour savoir quelles tâches devraient exclusivement être soumises à l'expertise médicale et lesquelles pourraient mieux être accomplies avec l'aide d'autres groupes professionnels.

Rechtslage oder auf einen Grundkonsens in unserer Gesellschaft, dass Sterbehilfe nicht erlaubt sein sollte, zu rechtfertigen. Die Situation ist für die Ärzteschaft um so schwieriger, als die Medien, die Politik und die Rechtsorgane oft wie selbstverständlich davon ausgehen, dass Sterbehilfe eine ärztliche Aufgabe sei. Um zu vermeiden, dass man von der Politik schliesslich einfach vor vollendete Tatsachen gestellt wird, ist die Ärzte- 
schaft herausgefordert, entweder in einer für die Gesellschaft nachvollziehbaren Weise zu begründen, warum Sterbehilfe und Medizin unvereinbar seien, oder aber an der Ausarbeitung einer für sie adäquaten Rolle in diesem Bereich teilzunehmen.

\section{Methode}

Für diese Untersuchung wurden sowohl Länder ausgewählt, in denen Sterbehilfe gesetzlich legalisiert oder aufgrund einer vorbestehenden offenen Rechtslage legal praktiziert wird (Belgien, Niederlande, Schweiz), als auch solche, in denen Sterbehilfe weiterhin illegal ist oder aus anderen Gründen aus der ärztlichen Praxis verbannt ist (Deutschland, Grossbritannien, Norwegen). Je ein Experte aus jedem dieser Länder verfasste einen Bericht zu folgenden Fragen:

1. Wie ist die Rechtslage zu Suizidbeihilfe und/ oder aktiver Sterbehilfe auf Verlangen im entsprechenden Land? Hat sich diese Rechtslage in den letzten Jahren verändert?

2. Welches ist die offizielle Position der Ärzteschaft zur Sterbehilfe und zu ihrer Rolle darin? Hat sich diese Position in den letzten Jahren verändert?

\section{Resultate}

\section{Sterbehilfe - Rechtslage}

\section{(Tab. 1, Kolonne links)}

Obwohl sowohl Suizidbeihilfe wie auch aktive Sterbehilfe auf Verlangen in den Niederlanden bis vor kurzem grundsätzlich illegal waren (Artikel 293 und 294 des niederländischen Strafgesetzbuches), wurden diese Handlungen, wenn sie von Ärzten unter Einhaltung gewisser Bedingungen praktiziert wurden, von den Gerichten seit den frühen 1970er Jahren toleriert. Im April 2002 dann wurden die Niederlande das erste Land der Welt, in dem Ärzte formell durch ein Gesetz von der Strafbarkeit der aktiven Sterbehilfe auf Verlangen ausgenommen wurden. Belgien, wo zuvor weder eine relevante Rechtsprechung im Einzelfall (case law) noch eine etablierte und regulierte Sterbehilfepraxis vorhanden war, folgte im September 2002 dem Nachbarland mit einem vergleichbaren, ausschliesslich die Ärzteschaft von der Strafbarkeit der aktiven Sterbehilfe auf Verlangen befreienden Gesetz nach.

In der Schweiz ist die Beihilfe zum Suizid, aber nicht die aktive Sterbehilfe auf Verlangen, gemäss dem auf das Jahr 1918 zurückgehenden Schweizerischen Strafgesetzbuch so lange nicht strafbar, als dem Sterbehelfer keine selbstsüchtigen Motive nachgewiesen werden können. Die
Straffreiheit der Suizidbeihilfe gilt für jeden Staatsbürger, Arzt oder Nichtarzt. Ähnlich ist die Rechtslage in Deutschland, wo ebenfalls die Beihilfe zum Suizid, nicht aber die aktive Sterbehilfe auf Verlangen grundsätzlich straffrei ist. Allerdings könnte hier, anders als in der Schweiz, die Beihilfe zum Suizid (bzw. das Unterlassen von Rettungsbemühungen, sobald der Sterbewillige das Bewusstsein verloren hat) strafrechtlich eventuell als unterlassene Hilfeleistung (Verletzung der sogenannten Garantenpflicht) beurteilt werden.

Sowohl die Beihilfe zum Suizid wie auch die aktive Sterbehilfe auf Verlangen sind gemäss dem norwegischen Strafgesetzbuch (Artikel 235 und 236), das auf das Jahr 1902 zurückgeht, verboten. Dasselbe gilt für Grossbritannien, wo selbst der (nichtassistierte) Suizid bzw. der Versuch dazu einen Straftatbestand darstellte, bis die sogenannte «Suicide Act» von 1961 die Strafbarkeit auf die Beihilfe und Verleitung zum Suizid durch Dritte beschränkte.

\section{Sterbehilfe - ärztlich-standesethische Richtlinien (Tab. 1, Kolonne rechts)}

Bei der Entwicklung des niederländischen Sterbehilfemodells spielte die niederländische Ärztegesellschaft von Anfang an eine Schlüsselrolle. Besorgnisse, dass die Sterbehilfe mit der ärztlichen Rolle in Konflikt stehe, standen nicht im Vordergrund. Auch Nichtärzten eine Mitverantwortung in diesen Entscheidungen zukommen zu lassen wurde nie ernsthaft in Betracht gezogen. Konsequenterweise kam kürzlich eine Arbeitsgruppe unter der Leitung der niederländischen Ärztegesellschaft, die zur Frage der Euthanasie bei Personen, die gar nicht krank sind, aber dennoch um Sterbehilfe ersuchen, zum Schluss, dass auch in diesen Fällen Ärzte die einzigen Experten sein sollten, um zu entscheiden, ob das «Leiden am Leben» stark genug sei, dass dem Ersuchen nachgekommen werden sollte.

Im Gegensatz zu den Niederlanden tat sich der belgische Rat der Nationalen Ärztekammer schwer damit, eine adäquate eigene Position zur Frage der Sterbehilfe zu erarbeiten, als er mit den schnellen und radikalen gesetzlichen Veränderungen in diesem Bereich in Belgien in den ersten Jahren dieses Jahrzehnts konfrontiert wurde. Artikel 95 der Verordnungen zur ärztlichen Berufsethik, der bisher den Ärzten die Beteiligung an Suizidbeihilfe oder aktiver Sterbehilfe in jeder Form untersagte, wurde sogar erst im März 2006 geändert. Gemäss dem revidierten Text dieser Verordnungen soll der Arzt den Patienten, dessen Lebensende naht, über die 


\section{Tabelle 1}

Sterbehilfe in sechs europäischen Ländern: gegenwärtige Rechtslage und standesethische Position (fettgedruckt) sowie Entwicklungen seit 2000 (kursiv).

\begin{tabular}{lll} 
& $\begin{array}{l}\text { Rechtslage zum assistierten Suizid (AS) und/ } \\
\text { oder zur aktiven Sterbehilfe auf Verlangen (E) } \\
\text { verboten } \rightarrow \text { AS/E erlaubt }\end{array}$ & $\begin{array}{l}\text { Ärztlich-standesethische Position zum assistierten Suizid (AS) und/ } \\
\text { oder zur aktiven Sterbehilfe auf Verlangen (E) } \\
\text { verboten } \rightarrow \text { AS/E neutral }\end{array}$ \\
\hline $\begin{array}{l}\text { Belgien } \\
\text { Deutschland }\end{array}$ & E verboten & AS/E verboten \\
\hline Niederlande & toleriert $\rightarrow$ AS/E erlaubt & AS/E erlaubt \\
\hline Norwegen & AS/E verboten & AS/E verboten \\
Schweiz & AS erlaubt & verboten $\rightarrow$ AS neutral \\
\hline Grossbritannien & E verboten & E verboten \\
& AS/E verboten & AS/E verboten $\leftrightarrow$ neutral
\end{tabular}

Schritte informieren, die unternommen werden können, wie die Wahl eines Bevollmächtigten, die Verweigerung, einem bestimmten Eingriff zuzustimmen, oder eine Willenserklärung zur Euthanasie. Speziell erwähnt wird die Pflicht des Arztes, den Patienten «rechtzeitig und deutlich darüber zu informieren, welchen Beistand er ihm während der Periode seines zu Ende gehenden Lebens zu leisten bereit ist», wobei der Patient über die erforderliche Zeit verfügen müsse, um einen zweiten ärztlichen Rat einzuholen.

Eine andere Form von «neutraler Haltung» gegenüber der Sterbehilfe hat die Schweizerische Akademie der Medizinischen Wissenschaften SAMW gewählt. In den neuen medizinisch-ethischen Richtlinien zur Betreuung von Patienten am Lebensende hält die SAMW zwar am grundsätzlichen Widerspruch zwischen Sterbehilfe und der ärztlichen Rolle fest («Beihilfe zum Suizid ist kein Teil der ärztlichen Tätigkeit»), respektiert im Gegensatz zu früher aber die Beihilfe zum Suizid als eine persönliche Entscheidung des Arztes im Einzelfall.

Demgegenüber verurteilen die ärztlichen Standesorganisationen in Deutschland, Norwegen und Grossbritannien die Sterbehilfe nach wie vor in jeder Form. Wie kontrovers aber auch in diesen Ländern die Thematik innerhalb der Ärzteschaft ist, zeigt die Tatsache, dass in Grossbritannien vor dem Hintergrund der Diskussion um die sogenannte «Assisted Dying for the Terminally Ill Bill», die 2005 im britischen Oberhaus eingebracht worden war, sowohl die British Medical Association wie auch das Royal College of Physicians of London für eine gewisse Zeit eine neutrale Position einnahmen. Später, als der Gesetzesvorschlag durch das Oberhaus abgelehnt worden war, krebsten beide Organisationen wieder zurück und nahmen erneut eine ablehnende Haltung ein.

\section{Diskussion}

\section{Die Ärzteschaft zwischen Widerstand und Sichdreinschicken}

Konfrontiert mit einer zunehmenden Befürwortung von Sterbehilfe in der Bevölkerung, mit zahlreichen politischen Vorstössen zur Öffnung des Strafrechts und bisher einigen wenigen effektiven Gesetzesänderungen, versuchen die meisten europäischen ärztlichen Standesorganisationen, eine Öffnung in diesem Bereich zu verhindern oder wenigstens hinauszuzögern.

Was effektiv stattfindet, könnte als eine Art Machtkampf beschrieben werden: hier eine Gesellschaft, die die Option ärztlicher Sterbehilfe verfügbar haben will, dort die ärztlichen Standesorganisationen, die Sterbehilfe als unvereinbar mit der eigenen Standesethik bezeichnen. Allerdings ist auch innerhalb der Ärzteschaft die ablehnende Haltung gegenüber der Sterbehilfe nicht einhellig. Sie ist nämlich generell am ablehnendsten bei denjenigen Spezialisten (wie beispielsweise den Onkologen oder Palliativmedizinern), die am wahrscheinlichsten diejenigen wären, die für solche Entscheidungen verantwortlich wären, sollte Sterbehilfe eine akzeptierte medizinische Praxis werden.

In Deutschland, Norwegen und Grossbritannien konnte die offizielle Ärzteschaft bis heute eine Öffnung in diesem Bereich verhindern. Immerhin war in Grossbritannien die Kampagne für die sogenannte «Assisted Dying for the Terminally Ill Bill» derart einflussreich, dass sie die Ablehung der British Medical Association und des Royal College of Physicians of London kurzfristig ins Wanken bringen konnte. Und in Deutschland ist die Bundesärztekammer mit ihrer strikten Ablehnung der ärztlichen Suizidbeihilfe in jüngster Zeit durch eine einflussreiche Gruppe von Juristen unter Druck geraten, die 
vorgeschlagen hat, dass Ärzte, die einem Patienten beim Suizid assistieren, weder strafrechtlich noch standesethisch belangt werden sollten.

In der Schweiz und in Belgien dagegen hat die Ärzteschaft in den letzten Jahren ihre Fundamentalopposition gegen die Sterbehilfe aufgegeben. Dieser Prozess war besonders eindrücklich in Belgien, wo die Ärzteschaft von den politischen Entwicklungen förmlich überrannt und mit dem neuen Sterbehilfegesetz mehr oder weniger vor ein fait accompli gestellt wurde. Schliesslich konnte der belgische Rat der Nationalen Ärztekammer nur noch konstatieren: «Wenn in einem demokratischen Staat ein Gesetz erlassen wird, und dieses Gesetz respektiert die Gewissensfreiheit des einzelnen Arztes, dann kann die Existenz eines solchen Gesetzes durch eine öffentliche Institution wie den belgischen Rat der Nationalen Ärztekammer nicht einfach ignoriert werden.»

Demgegenüber bestand in den Niederlanden der 80er und 90er Jahre eine weitgehende Symmetrie zwischen der Rolle, die die Rechtspraxis, und der Rolle, die die niederländische Ärztegesellschaft sich selber bei der Euthanasie zuschrieb. Allerdings zeigt die Entwicklung der letzten Jahre in den Niederlanden nicht nur eine anhaltende Unwilligkeit der Ärzte, Fälle von geleisteter Euthanasie den Behörden zu melden, sondern auch eine Rückkehr zu Praktiken, wie zum Beispiel der sogenannten terminalen Sedierung, die als normale medizinische Massnahmen am Lebensende akzeptiert sind und bei denen auch keine Pflicht zur Meldung an die Behörden besteht.

\section{Ärzte als exklusive Sterbehilfeexperten?}

Die offene gesetzliche Regulierung von Sterbehilfe bringt die Ärzte in einen grundsätzlichen Konflikt. Auf der einen Seite möchten sie sich aus der Thematik möglichst heraushalten, auf der anderen Seite, wenn eine Gesetzesöffnung unvermeidlich erscheint, möchten sie wenigstens bestimmen können, in welchen Fällen Sterbehilfe erlaubt sein sollte und in welchen nicht.

Je mehr die Ärzteschaft sich aber an diesen Diskussionen beteiligt, desto mehr gerät sie unfreiwillig in die Rolle von Experten in einem Bereich, der tatsächlich weit über die Medizin hinausgeht. Und genau auf dieser exklusiven Expertenrolle für Sterbehilfe möchten so manche Sterbehilfegesetze oder -gesetzesvorschläge die Ärzteschaft noch so gerne fixieren.

Eine uneingeschränkte Annahme dieser Rolle würde letztlich nichts anderes als die Einführung des niederländischen Sterbehilfemodells bedeu- ten, in dem Suizidbeihilfe und aktive Sterbehilfe auf Verlangen als weitere «medizinische Entscheidungen am Lebensende» neben der passiven und der indirekten Sterbehilfe zur akzeptierten Praxis geworden sind. Diese exklusive Expertenrolle für die Ärzteschaft erscheint allerdings um so störender, je mehr, wie das in den Niederlanden der Fall war, die «Indikationen» für die Sterbehilfe zunehmend ausgeweitet werden. Die Frage der Sterbehilfe aufgrund eines reinen «Leidens am Leben» rührt an ganz persönlichphilosophische wie auch an bedeutsame soziale Fragen. Da aber in diesen Fällen gar nichts eigentlich Medizinisches vorliegt, ist nicht einzusehen, warum Ärzte in solchen Fällen mehr Entscheidungskompetenz haben sollten als irgendeine andere Berufsgruppe.

So erstaunt es denn auch nicht, dass in den letzten Jahren zunehmend der Vorschlag aufgetaucht ist, dass die Sterbehilfe als Dienstleistung ausserhalb der medizinischen Versorgung anzusiedeln sei. Nicht klinisch tätige Ärzte, sondern ein interdisziplinär zusammengesetztes Team wäre dafür verantwortlich, und ein entsprechendes Gesetz müsste denn auch nicht die Ärzteschaft als Ganzes, sondern vielmehr dieses Expertenteam von der generellen Strafbarkeit der Sterbehilfe befreien. Ein solches Modell könnte sicherstellen, dass die Eignung der sterbewilligen Personen zur Sterbehilfe kompetent abgeklärt würde nach Kriterien, die zuvor in einem politischen Prozess transparent festgelegt worden wären. Zudem gäbe es für die betreuenden Ärzte keinerlei Rollenkonflikte, indem ihre Rolle klar darauf beschränkt wäre, mit ihren Patienten die Situation offen zu diskutieren, Möglichkeiten der Behandlung inklusive aller palliativmedizinischen Optionen $\mathrm{zu}$ erörtern und dabei jede mögliche Unterstützung anzubieten.

Dieses Modell würde eine vermehrte Verantwortungsübernahme des Staates in der Sterbehilfe verlangen. Die Erfahrung zeigt allerdings, dass das staatliche Interesse, sich in einem Bereich zu engagieren, in dem es extrem schwierig ist, geeignete Entscheidungskriterien zu entwickeln, und in dem gleichzeitig jede falsche Entscheidung weitreichende und irreversible Konsequenzen hat, sich in engen Grenzen hält.

\section{Ein interdisziplinärer Ansatz?}

Wenn eine Gesellschaft die Option der Sterbehilfe verfügbar machen will, so sollte die Verantwortung für diese Entscheidungen so breit wie möglich abgestützt werden. Es ist nicht damit getan, dass Gesetze und ethische Richtlinien die Grenzen festlegen, innerhalb deren Ärzte Sterbe- 
hilfe leisten dürfen, und dass die Einhaltung dieser Kriterien im nachhinein von Juristen und wie dies in den Niederlanden der Fall ist - von Ethikern geprüft wird. Vielmehr sollte diesen beiden Berufsgruppen, zusammen mit anderen Experten, wie Theologen/Pfarrern, Pflegenden, Apothekern, Sozialarbeitern, und überhaupt jeder Art von medizinischen «Laien» mit genügend Lebenserfahrung, die Möglichkeit gegeben werden, Mitverantwortung in spezifischen Fällen zu tragen. Eine andere Frage ist es, ob ein solcher interdisziplinärer Ansatz am besten innerhalb eines vom Staat betriebenen «Sterbehilfeservice» verwirklicht werden könnte, wie dieser oben skizziert wurde. Ein solches Modell dürfte wohl zu bürokratisch und unpersönlich sein, um den Bedürfnissen der um Sterbehilfe ersuchenden Personen und ihrer Angehörigen gerecht zu werden.

Auf alle Fälle ist es in diesem Stadium der Diskussion eine wichtige Aufgabe der Ärzteschaft, sorgfältig zu erörtern, wo in diesem Bereich medizinische Expertise unersetzbar ist und wo diese nicht weiterhelfen kann. Dies ist keine selbstverständliche Forderung angesichts der Tatsache, dass noch immer die meisten Ärztevereinigungen es ablehnen, überhaupt in diese Diskussion einzutreten. Es gibt allerdings auch andere Beispiele. In den USA gelang es bereits in den späten 1990er Jahren dem Bioethikzentrum der Universität von Pennsylvania, eine Anzahl anerkannter Experten an einem runden Tisch zu versammeln. Diese Arbeitsgruppe kam zum Schluss, dass im Vorfeld einer Abklärung eines Verlangens nach Sterbehilfe die Information des Patienten über dessen Diagnose, Prognose sowie über die verschiedenen Behandlungsmöglichkeiten eindeutig ärztliche Aufgabe sei. Konkomittierende Faktoren wie beispielsweise eine Depression müssten ebenfalls notwendigerweise durch einen Arzt abgeklärt werden. Die Abklärung einer möglichen Fremdbeeinflussung, spirituelle Fragen, ja sogar eine adäquate Symptomkontrolle sei dagegen in den Händen von Pflegenden, Sozialarbeitenden oder von Seelsorgern besser aufgehoben. Die Expertengruppe warf auch die Frage auf, ob es nicht besser sei, die Koordination und Überwachung des gesamten möglicherweise zum assistierten Tod führenden Prozesses einer nichtärztlichen Fachperson zu überlassen.
Zwischen den Schlüssen dieser Expertengruppe und den aktuellen medizinisch-ethischen Richtlinien der Schweizerischen Akademie der Medizinischen Wissenschaften zur Betreuung von Patienten am Lebensende finden sich interessante Parallelen, obwohl die beiden Texte unabhängig voneinander vor dem Hintergrund ganz verschiedener Gesundheitssysteme und eines ganz unterschiedlichen kulturellen und rechtlichen Kontexts entstanden sind. Auch die Richtlinien der SAMW identifizieren nämlich zum einen die Feststellung des nahenden Lebensendes (Diagnose/Prognose), zum anderen das Angebot alternativer Möglichkeiten der Hilfestellung (Palliative Care) als spezifisch medizinische Verantwortlichkeit bei der Suizidbeihilfe. Auf der anderen Seite halten sie ausdrücklich fest, dass die Feststellung der Urteilsfähigkeit, des Vorliegens eines konstanten und wohlerwogenen Sterbewunsches sowie der Absenz äusseren Drucks auf die sterbewillige Person nicht exklusiv durch ärztliche Expertise erfolgen muss, so dass die von der SAMW geforderte Zweitmeinung in diesen Punkten nicht zwingend durch einen Arzt zu erfolgen habe.

\section{Schlussfolgerungen}

Vor dem Hintergrund einer zunehmenden Befürwortung von Sterbehilfe in der Öffentlichkeit europäischer Länder bleibt die entscheidende Frage nach der adäquaten Rolle des Arztes in der Sterbehilfe umstritten. Eine Gesellschaft, die Sterbehilfe grundsätzlich zulassen will, sollte sich sorgfältig überlegen, welche Aufgaben in diesem Zusammenhang ausschliesslich ärztlicher Expertise überlassen werden sollten und welche Aufgaben durch den Einbezug anderer Berufsgruppen besser wahrgenommen werden könnten.

\section{Literatur}

Ausführliche Literaturangaben finden sich in dem diesem Text zugrundeliegenden Originalartikel:

- Bosshard G, Broeckaert B, Clark D, Materstvedt LJ, Gordijn B, Müller-Busch HC. A role for doctors in assisted dying? An analysis of legal regulations and medical professional positions in six European countries. J Med Ethics. 2008;34:28-32. http:// jme.bmj.com/cgi/content/full/34/1/28. 